\title{
Correction to: On the Continuity of the Tangent Cone to the Determinantal Variety
}

\author{
${\text { Guillaume } \text { Olikier }^{1} \text {-P.-A. Absil }}^{1}$ \\ Published online: 16 February 2022 \\ (C) Springer Nature B.V. 2022
}

\section{Correction to: Journal of Physiology and Biochemistry https://doi.org/10.1007/s11228-021-00618-9}

The original version of this article unfortunately contained an incorrect in-text data. Author has sent the corrections via email for this to be rectified accordingly.

The corrections are shown below.

(1) In the entire document, none of the hyperref links towards the theorem, proposition, lemma, and corollary environments is working.

(2) The figure is a screenshot. The font is not the same as the one used for the text. The figure must be compiled with the TikZ package.

(3) The table does not have the same design as the one in the manuscript. The first horizontal line is too long and the vertical lines are missing.

(4) The figure and the table are not well positioned. They should be positioned using the [h] command.

(5) There is no full stop "." at the end of the captions of both the figure and the Table.

(6) The smallmatrix command has not been used properly. This appears in Section 3 and in Section 4.1.

(7) The Vlimits command has not been used properly in eqs. (30) and (31), and in the two displayed equations following eq. (33). Moreover, in the second equation following eq. (33), the " $\&$ " command in the "align*" environment has not been used properly.

(8) In the first sentence of Section 4.1, "(5), (6)" must be replaced by "(5) and (6)".

(9) In the displayed equation of Proposition 5.2, the "\&\&" space in the "align*" environment does not appear.

(10) In the subsections of Section 5, m, n, and $\mathrm{r}$ in $\backslash \operatorname{mathbb}\{\mathrm{R}\}_{-}\{\backslash \mathrm{le} \mathrm{r}\}^{\wedge}\{\mathrm{m} \backslash$ times $\mathrm{n}\}$ appear in bold in the titles. They should be written in regular font.

Moreover, we would like the following typo to be corrected.

The online version of the original article can be found at https://doi.org/10.1007/s11228-021-00618-9

Guillaume Olikier

guillaume.olikier@uclouvain.be

1 ICTEAM Institute, UCLouvain, Avenue Georges Lema^1tre 4, 1348 Louvain-la-Neuve, Belgium 
(11) In the proof of Proposition 4.1, in the penultimate sentence and in the preceding one (thus, at two places), "\$r_i - lushort $\{r\} \$$ first columns" must be replaced by "first $\$ r \_i-l u s h o r t\{r\} \$$ columns".

Publisher's Note Springer Nature remains neutral with regard to jurisdictional claims in published maps and institutional affiliations. 